\title{
Construction of therapeutically relevant human prostate epithelial fate map by utilising miRNA and mRNA microarray expression data
}

\begin{abstract}
Jayant K Rane ${ }^{1,7}$, Antti Ylipää ${ }^{2,3}$, Rachel Adamson ${ }^{1}$, Vincent M Mann ${ }^{4,5}$, Matthew S Simms ${ }^{4,5}$, Anne T Collins ${ }^{1}$, Tapio Visakorpi ${ }^{6}$, Matti Nykter ${ }^{2,3}$ and Norman J Maitland ${ }^{\star, 1,5}$

${ }^{1}$ Department of Biology, YCR Cancer Research Unit, University of York, York YO10 5DD, North Yorkshire, UK; ${ }^{2}$ Prostate Cancer Research Center, Institute of Biosciences and Medical Technology (BioMediTech), University of Tampere and Tampere University Hospital, Biokatu 8, Tampere 33520, Finland; ${ }^{3}$ Department of Signal Processing, Tampere University of Technology, Korkeakoulunkatu 10, Tampere 33720, Finland; ${ }^{4}$ Hull York Medical School, University of Hull, Hull HU6 7RX, East Yorkshire, UK; ${ }^{5}$ Department of Urology, Castle Hill Hospital, Cottingham HU16 5JQ, East Yorkshire, UK and ${ }^{6}$ Molecular Biology of Prostate Cancer Group, Institute of Biosciences and Medical Technology (BioMediTech), University of Tampere and Tampere University Hospital, BioMediTech, Biokatu 8, Tampere 33520, Finland
\end{abstract}

Background: Objective identification of key miRNAs from transcriptomic data is difficult owing to the inherent inconsistencies within miRNA target-prediction algorithms and the promiscuous nature of miRNA-mRNA target relationship.

Methods: An integrated database of miRNAs and their 'relevant' mRNA targets was generated from validated miRNA and mRNA microarray data sets generated from patient-derived prostate epithelial normal and cancer stem-like cells (SCs) and committed basal (CB) cells. The effect of miR-542-5p inhibition was studied to provide proof-of-principle for database utility.

Results: Integration of miRNA-mRNA databases showed that signalling pathways and processes can be regulated by a single or relatively few miRNAs, for example, DNA repair/Notch pathway by miR-542-5p, $P=0.008$. Inhibition of miR-542-5p in CB cells (thereby achieving miR-542-5p expression levels similar to SCs) promoted efficient DNA repair and activated expression of Notch reporters, HES1 and Survivin, without inducing dedifferentiation into SCs.

Conclusions: Our novel framework impartially identifies therapeutically relevant miRNA candidates from transcriptomic data sets.

There are $<2000$ known human miRNAs, which influence the expression of at least $60 \%$ of cell proteins in a tissue-specific manner (Friedman et al, 2009), achieving precise orchestration of cell fate decisions. Sequencing of whole genomes, fractionated hierarchical sub-populations, and utilisation of sophisticated computational algorithms have revealed that miRNAs could be exploitable as both biomarkers and therapeutic targets for a number of diseases, particularly cancers (Catto et al, 2011; Iorio and Croce, 2012). However, objective identification and functional validation of candidate miRNAs remain difficult, owing to inherent inconsistencies with miRNA target-prediction algorithms and the promiscuous nature of miRNA-mRNA target relationship.

\footnotetext{
*Correspondence: Professor NJ Maitland; E-mail: n.j.maitland@york.ac.uk

${ }^{7}$ Current address: Leukaemia and Stem Cell Biology Group, Department of Haematological Medicine, King's College London, Rayne Institute, 123 Coldharbour Lane, Denmark Hill, London SE5 9NU, UK
}

Received 15 April 2015; revised 8 June 2015; accepted 11 June 2015; published online 23 July 2015 
To identify cell type-specific biomarker/therapeutically relevant miRNAs in prostate cancer, we have integrated miRNA (Rane et al, 2015) and mRNA (Birnie et al, 2008) microarray data sets. These data sets profiled miRNA and mRNA expression from patientderived, human prostate stem-like cells (SC-CD $\left.133^{+} / \alpha_{2} \beta_{1}^{\text {hi }}\right)$ and their differentiated progeny: committed basal (CB-CD133- $/ \alpha_{2} \beta_{1}^{\text {lo }}$ ) cells (Figure 1A).

\section{MATERIALS AND METHODS}

Sample procurement. Patient prostate tissue samples were obtained after written consent and full ethical approval (LREC 07/H1304/121). Tissue collection and epithelial cell culture were performed as described before (Collins et al, 2005; Rane et al, 2014).

miRNA-mRNA microarray data integration. The details of samples, methods and the platform used for miRNA (accession code: GSE59156) and mRNA (accession code: E-MEXP-993) microarray expression database generation were described before (Birnie et al, 2008; Rane et al, 2015). The co-expression analysis was performed using Pearson correlation as the distance metric and Ward's linkage method. miRNA target prediction was performed using eight different prediction algorithms on the miRWalk website (http://www.umm.uni-heidelberg.de/apps/zmf/ mirwalk/; Lewis et al, 2005; Dweep et al, 2011). Target mRNAs not inversely expressed in the appropriate homologous cell type were removed. The pathway enrichment scores were computed on the inversely expressed mRNAs using standard hypergeometric tests. For more details see Supplementary File S1.

Expression analysis. miRVana kit (Life Technology, Paisley, UK) was used to extract RNA. miRNA expression was assessed with miScript primer assays (Qiagen $\mathrm{GmbH}$, Hilden, Germany). mRNA expression was assessed by qRT-PCR and normalised first to RPLP0 and then with control expression using respective TaqMan probes (Life Technology). Immunofluorescence studies were performed as described before (Frame et al, 2013). For details of antibodies used, see Supplementary Table S1. Nuclear $\gamma$-H2AX foci and BRCA1/RAD51 positive nuclei were counted manually.

Transfection of miRNA inhibitor. CB cells were transfected with $100 \mathrm{~nm}$ miScript inhibitor for miR-542-5p (miR-542-5p-i) or miScript inhibitor negative control (Qiagen $\mathrm{GmbH}$ ) using viromer BLUE (Lipocalyx GmbH, Halle (Saale), Germany) for $72 \mathrm{~h}$ according to the manufacturer's protocol.

Irradiation of cells and colony-forming efficiency. Irradiation of cells, and colony-forming efficiency were described previously (Rane et al, 2015).

Statistical analysis. All experiments were performed on $3 \mathrm{BPH}$ and $3 \mathrm{PCa}$ samples from individual patients. Errors are the standard deviation of mean. The significance was determined using Student's two-tailed $t$-test, ${ }^{\star} P<0.05,{ }^{* \star} P<0.01,{ }^{* * *} P<0.001$.

\section{RESULTS}

miRNA-mRNA microarray database integration. To integrate the miRNA and mRNA microarray data sets, we firstly leveraged the observation that expressed levels of functionally relevant miRNAs and their target mRNAs should be inversely correlated, as mammalian miRNAs are known to suppress not only protein expression, but also the transcript levels of their targets (Guo et al, 2010). Having identified differentially expressed miRNAs in SC vs $\mathrm{CB}$ cells $(P<0.05)$, the targets for each miRNA were selected based on positive target-prediction call by at least three prediction

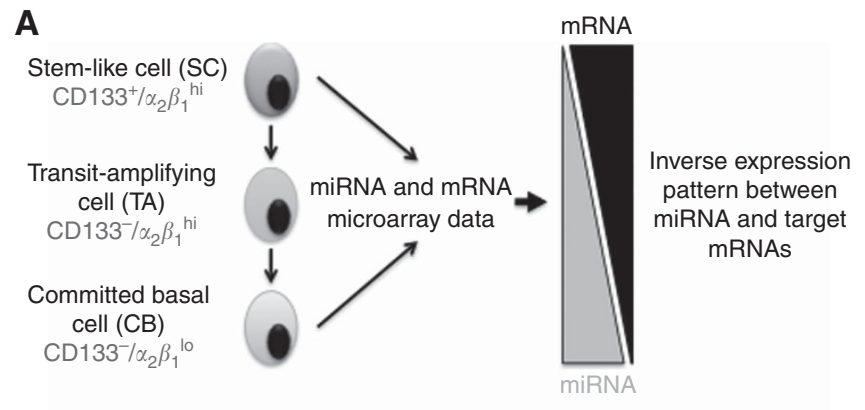

B

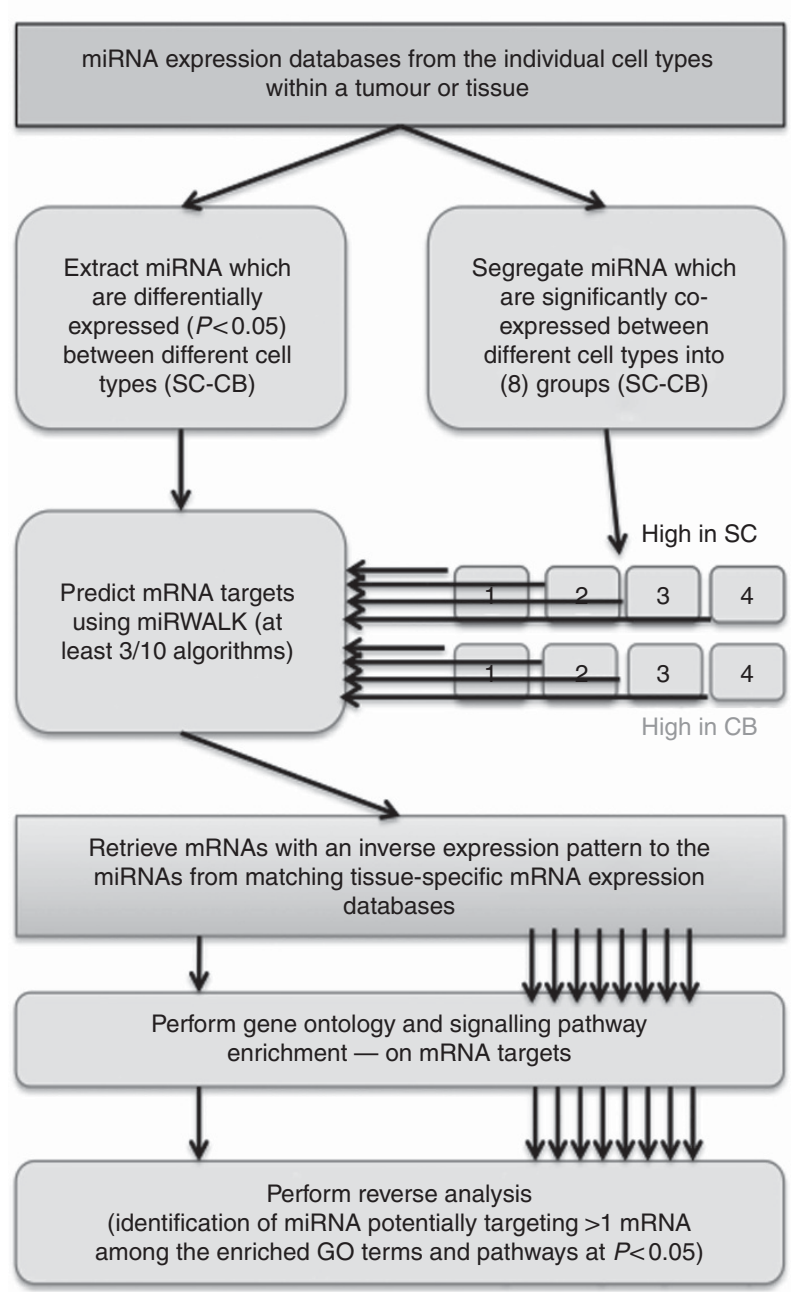

Figure 1. Generation of integrated miRNA-mRNA data sets using expression data from prostate SC and CB cells. (A) A schematic of human prostate epithelial hierarchy (left) showing a stem-like cell (SC) with a basal phenotype differentiating into committed basal (CB) cell via transit-amplifying (TA) cell. The nature of inverse expression relationship between miRNA and its target mRNAs is depicted on the right side. For the purpose of data integration, only the expression profiles from SC and CB cells were used. (B) Algorithm used to generate integrated miRNA-mRNA data set. Two approaches were taken: one without co-expression analysis on miRNA data and second with co-expression analysis. The eight sub-groups in co-expression analysis were kept separate at each subsequent step in the analysis.

algorithms and on an inverse expression pattern (Figure 1A and B). The analysis implied that signalling pathways/processes (e.g. DNA repair associated with $\mathrm{miR}-542-5 \mathrm{p} / 99 \mathrm{a} / 100, P<0.01$ ) were regulated by a single or relatively few miRNAs, each of which affected multiple genes associated with the same pathway 
(Supplementary File S2). To investigate whether diverse miRNAs can have similar pathway-regulating functions, we employed a second approach, where differentially expressed miRNAs (SC vs $\mathrm{CB}$ ) were K-means clustered into eight distinct sets of coexpressed miRNAs prior to re-running the previous analysis for each cluster separately (Figure 1B and Supplementary File S3). Such co-expressed clusters were expected to have coordinated functions, which should be evident through their targets and their seed-sequence similarity (Lee et al, 2004; Supplementary File S4). The first evidence in support of this hypothesis was the observation that miRNAs with known functional associations (e.g., let-7 miRNAs and miRNAs of miR-17-92 family clustered within the same sub-group; Supplementary File S3). Notch signalling has been shown to have a significant role in prostate cancer cell fate (Carvalho et al, 2014; Supplementary Figure S1) Therefore it was of interest to further investigate miR-542-5p (identified by both in silico analyses), which can regulate Notch pathway-associated protein MAML1 (Supplementary File S4).

Proof-of-principle experiments to validate miRNA-mRNA database predictions. To test the validity of in silico predictions from the above two approaches, we measured the functional impact of miR-542-5p inhibition on prostate epithelial fate through

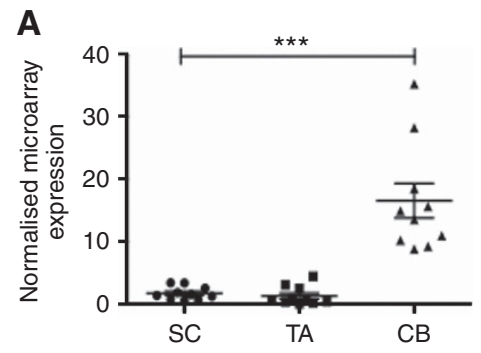

C

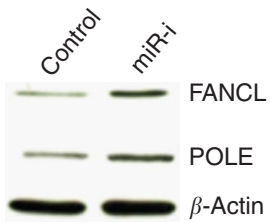

D

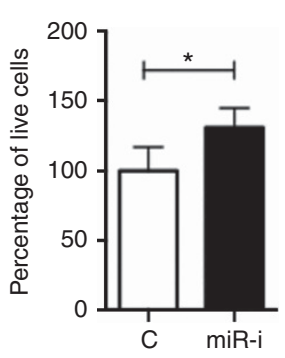

FANCL, POLE1 and MAML1. Little is known about the role of miR-542-5p in prostate cancers, but its predicted targets, FANCL (Rajendra et al, 2014) and POLE1 (Palles et al, 2013), are vital mediators of DNA repair, whereas MAML1 is a critical transcriptional co-activator of Notch receptors that stimulates transcription of Hes1 (Wu et al, 2000). We have previously shown that the SCs from normal prostate and malignant tissues are relatively radioresistant (Frame et al, 2013); however, they can be sensitised to radiation by concurrent inhibition of Notch signalling by the gamma-secretase inhibitor RO4929097 (Tolcher et al, 2012; Supplementary Figure S1). As miR-542-5p is suppressed in SC (compared with $\mathrm{CB}$; Figure $2 \mathrm{~A}$ and $\mathrm{B}$ ), we hypothesised that this lower expression of miR-542-5p in SCs contributes to radioresistance. Inhibition of miR-542-5p expression in CB cells (Supplementary Figure S2) resulted in elevated expression of POLE1 and FANCL (Figure 2C). We also observed better cellular recovery (Figure 2D), quicker $\gamma-\mathrm{H} 2 \mathrm{~A}$.X recovery (Figure $2 \mathrm{E}$ ) and more efficient BRCA1 and RAD51 foci formation (Figure $2 \mathrm{~F}$ and $\mathrm{G}$ ) after exposure of the $\mathrm{CB}$ cells to $5 \mathrm{~Gy}$ radiation. The efficient DNA repair was also accompanied by activation of Notch signalling as demonstrated by upregulation of MAML1 protein (1.8-fold) and Notch targets HES1 (2.1-fold) and Survivin (4.7-fold; Figure 2H). A similar trend was also observed at the
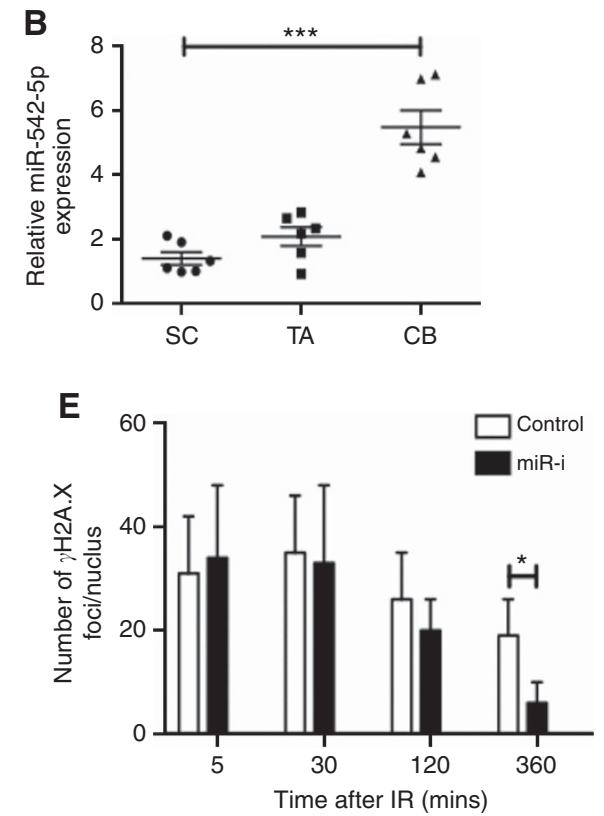

$\mathbf{F}$

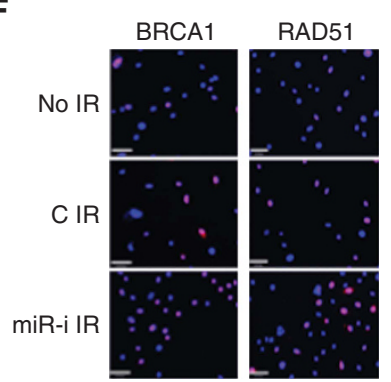

G

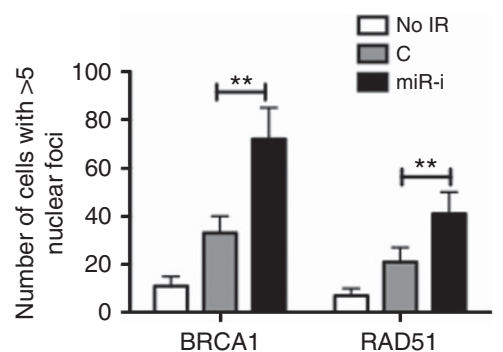

H

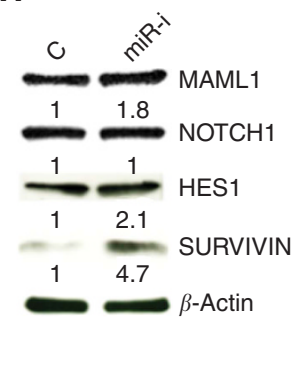

Figure 2. Proof-of-principle experiments to validate miRNA-mRNA database predictions using miR-542-5p. (A) miR-542-5p expression in miRNA microarray data set and (B) qRT-PCR validation on independent samples. CB cells were then transfected with 100 nm miR-542-5p-inhibitor ('miR-I') or negative control (' $\mathrm{C}$ ') for 3 days. The expression of FANCL and POLE proteins was assessed by western blot (C). These cells were irradiated with $5 \mathrm{~Gy}$ IR before counting live cell count after $48 \mathrm{~h}$ of IR (D), $\gamma$-H2AX immunofluorescence foci/nucleus recovery (E), BRCA1 and RAD51 nuclear positivity $2 \mathrm{~h}$ after IR (F) Blue: DAPI and lilac: BRCA1/RAD51, and quantification is shown in G. Protein expression changes in MAML1 and Notch-associated effector proteins HES1 and Survivin (H). Error bars $=60 \mu \mathrm{m}$. Each experiment represents mean of $3 \mathrm{BPH}$ and $3 \mathrm{PCa}$ and plotted as mean $\pm \mathrm{s} . \mathrm{d}$. ${ }^{\star} P<0.05,{ }^{\star \star} P<0.01,{ }^{* \star *} P<0.001$ (Student's t-test). Abbreviations: $\mathrm{SC}=$ stem-like cells; TA=transit-amplifying cells; $C B=$ committed basal cells; $\mathrm{BPH}=$ benign prostatic hyperplasia; $\mathrm{PCa}=$ treatment naive $\mathrm{Gl}-7$ prostate cancer; miR-I=miR-542-5p inhibitor; $\mathrm{C}=$ inhibitor negative control. 

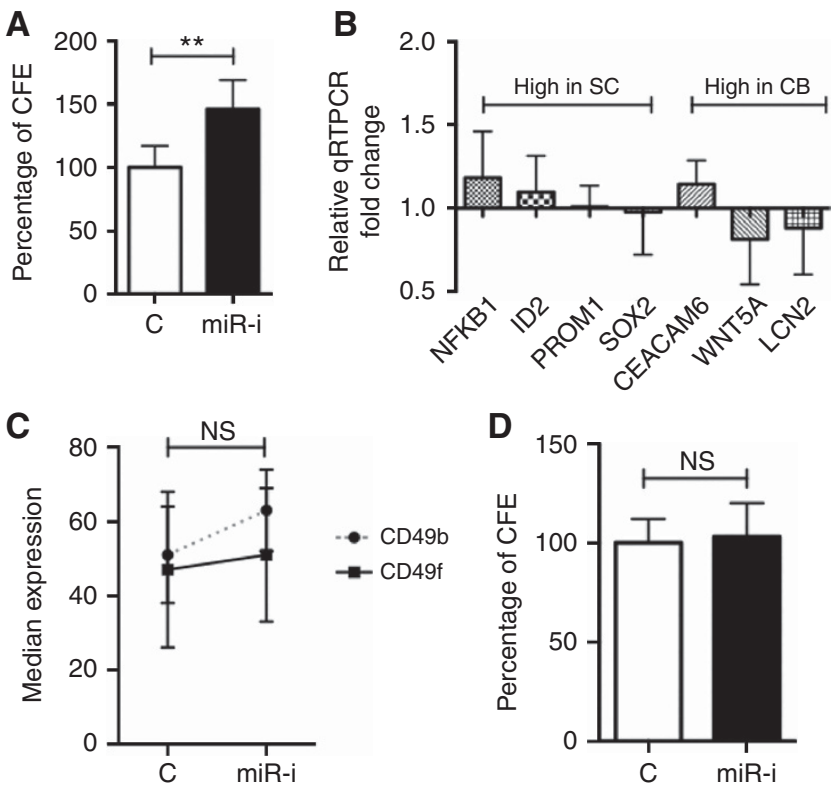

Figure 3. Effect of miR-542-5p inhibition on differentiation state of prostate epithelial cells. (A) Colony-forming efficiency (CFE) of miR542-5p inhibitor (or negative control)-transfected CB cells after exposure to $5 \mathrm{~Gy}$ radiation ( $n=3 \mathrm{BPH}$ and PCa each). Before radiation, $C B$ cells were transfected with miR-542-5p inhibitor for $72 \mathrm{~h}$. $\mathrm{CB}$ cells transfected with miR-542-5p inhibitor (or negative control) for $72 \mathrm{~h}$ and subjected to qRT-PCR analysis for known SC/CB associated genes (B), FACS analysis for CD49b/f (C) and colony-forming efficiency (D). Each experiment represents the mean of $3 \mathrm{BPH}$ and 3 PCa and plotted as mean \pm s.d. (except C). ${ }^{\star \star} P<0.01$ (Student's ttest). Abbreviations: $\mathrm{SC}=$ stem-like cells; $\mathrm{CB}=\mathrm{committed}$ basal cells; $\mathrm{BPH}=$ benign prostatic hyperplasia; $\mathrm{PCa}=$ treatment naive $\mathrm{Gl}-7$ prostate cancer; $\operatorname{miR}-\mathrm{I}=\mathrm{miR}-542-5 \mathrm{p}$ inhibitor; $\mathrm{C}=$ inhibitor negative control; NS=not significant.

mRNA level, especially for Notch effector HES1 and Survivin (Supplementary Figure S3).

Absence of a stem-like phenotype in miR-542-5p inhibited CB cells. A substantial (50\%) increase in colony-forming ability (Figure 3A) was also noted in miR-542-5p inhibited CB cells after radiation treatment $(5 \mathrm{~Gy})$. As we have shown that SCs are more radio-resistant than $\mathrm{CB}$ cells (Frame et al, 2013), the observed efficient DNA repair after miR-542-5p inhibition in CB cells could either be due to the direct effects of POLE1, FANCL and MAML1 overexpression, or the result of dedifferentiation of $\mathrm{CB}$ into SCs (or both). However, inhibition of miR-542-5p did not result in any detectable dedifferentiation of $\mathrm{CB}$ cells (Figure 3B-D). Thus, miR-542-5p inhibition in CB cells resulted solely in efficient DNA repair and Notch activation, as predicted by our miRNA-mRNA data integration data sets.

\section{DISCUSSION}

The central theme of this work was to devise a means for the identification of functionally relevant miRNAs and their target mRNAs. Our approach selects only tissue-specific mRNA targets in the specific cellular context, such as SC fate determination in present study. As the reduction in target mRNA expression may not always be evident (as we have assumed), our investigation may have underestimated the overall influence of miRNAs in prostate carcinogenesis. However, the causal relationship between some of the identified miRNA and their target pathways/ processes, for example, miR-99a/100 regulation of DNA repair (Mueller et al, 2013; Rane et al, 2015), clearly demonstrates the validity of our approach. Furthermore, the importance of studying miRNAs in a specific cellular phenotype has recently been underscored by the discovery that the expression changes in miR-143/145 in purified mouse colon stromal cells, rather than epithelial components (as previously assumed from unfractionated tissue/tumour biopsies) has functional relevance in regenerating mouse intestine (Chivukula et al, 2014). This pathway/process-based approach also provides an initial framework to address the long-standing issue of cooperative or functionally inter-related nature of miRNA targets in a practical setting. The database integration methods we have described can be readily adapted for any other tissue types to generate functionally relevant and therapeutically viable candidates, as exemplified here by miR-542-5p. In combination with alternative approaches such as large-scale miRNA functional screens, in an appropriate cellular environment (as we demonstrate), our approach should bring miRNA-driven therapeutics closer to direct clinical application.

\section{ACKNOWLEDGEMENTS}

We thank all the patients and urology surgeons L Coombes, G Cooksey and J Hetherington (Castle Hill Hospital, Cottingham, UK). We also thank Dr Davide Pellacani for useful discussions. The work was funded by PRO-NEST Marie-Curie Grant (to JKR), The Finnish Funding Agency for Technology and Innovation Finland Distinguished Professor programme, and Academy of Finland: project no. 132877 (to AY and MN), project support from Cancer Research Technology (to RA), and Yorkshire Cancer Research (to ATC and NJM).

\section{CONFLICT OF INTEREST}

The authors declare no conflict of interest.

\section{REFERENCES}

Birnie R, Bryce SD, Roome C, Dussupt V, Droop A, Lang SH, Berry PA, Hyde CF, Lewis JL, Stower MJ, Maitland NJ, Collins AT (2008) Gene expression profiling of human prostate cancer stem cells reveals a pro-inflammatory phenotype and the importance of extracellular matrix interactions. Genome Biol 9(5): R83.

Catto JW, Alcaraz A, Bjartell AS, De Vere White R, Evans CP, Fussel S, Hamdy FC, Kallioniemi O, Mengual L, Schlomm T, Visakorpi T (2011) MicroRNA in prostate, bladder, and kidney cancer: a systematic review. Eur Urol 59(5): 671-681.

Carvalho FL, Simons BW, Eberhart CG, Berman DM (2014) Notch signaling in prostate cancer: a moving target. Prostate 74(9): 933-945.

Chivukula RR, Shi G, Acharya A, Mills EW, Zeitels LR, Anandam JL, Abdelnaby AA, Balch GC, Mansour JC, Yopp AC, Maitra A, Mendell JT (2014) An essential mesenchymal function for miR-143/145 in intestinal epithelial regeneration. Cell 157(5): 1104-1116.

Collins AT, Berry PA, Hyde C, Stower MJ, Maitland NJ (2005) Prospective identification of tumorigenic prostate cancer stem cells. Cancer Res 65(23): 10946-10951.

Dweep H, Sticht C, Pandey P, Gretz N (2011) miRWalk-database: prediction of possible miRNA binding sites by "walking" the genes of three genomes. J Biomed Inform 44(5): 839-847.

Frame FM, Pellacani D, Collins AT, Simms MS, Mann VM, Jones GD, Meuth M, Bristow RG, Maitland NJ (2013) HDAC inhibitor confers radiosensitivity to prostate stem-like cells. Br J Cancer 109(12): 3023-3033.

Friedman RC, Farh KK, Burge CB, Bartel DP (2009) Most mammalian mRNAs are conserved targets of microRNAs. Genome Res 19(1): 92-105. 
Guo H, Ingolia NT, Weissman JS, Bartel DP (2010) Mammalian microRNAs predominantly act to decrease target mRNA levels. Nature 466(7308): 835-840.

Iorio MV, Croce CM (2012) MicroRNA dysregulation in cancer: diagnostics, monitoring and therapeutics. A comprehensive review. EMBO Mol Med 4(3): 143-159.

Lee HK, Hsu AK, Sajdak J, Qin J, Pavlidis P (2004) Coexpression analysis of human genes across many microarray data sets. Genome Res 14(6): 1085-1094.

Lewis BP, Burge CB, Bartel DP (2005) Conserved seed pairing, often flanked by adenosines, indicates that thousands of human genes are microRNA targets. Cell 120(1): 15-20.

Mueller AC, Sun D, Dutta A (2013) The miR-99 family regulates the DNA damage response through its target SNF2H. Oncogene 32(9): 1164-1172.

Palles C, Cazier JB, Howarth KM, Domingo E, Jones AM, Broderick P, Kemp Z, Spain SL, Guarino E, Salguero I, Sherborne A, Chubb D, CarvajalCarmona LG, Ma Y, Kaur K, Dobbins S, Barclay E, Gorman M, Martin L, Kovac MB, Humphray S. Consortium C, Consortium WGSLucassen A, Holmes CC, Bentley D, Donnelly P, Taylor J, Petridis C, Roylance R, Sawyer EJ, Kerr DJ, Clark S, Grimes J, Kearsey SE, Thomas HJ, McVean G, Houlston RS, Tomlinson I (2013) Germline mutations affecting the proofreading domains of POLE and POLD1 predispose to colorectal adenomas and carcinomas. Nat Genet 45(2): 136-144.
Rajendra E, Oestergaard VH, Langevin F, Wang M, Dornan GL, Patel KJ, Passmore LA (2014) The genetic and biochemical basis of FANCD2 monoubiquitination. Mol Cell 54(5): 858-869.

Rane JK, Droop AP, Pellacani D, Polson ES, Simms MS, Collins AT, Caves LS, Maitland NJ (2014) Conserved two-step regulatory mechanism of human epithelial differentiation. Stem Cell Rep 2(2): 180-188.

Rane JK, Scaravilli M, Ylipaa A, Pellacani D, Mann VM, Simms MS, Nykter M, Collins AT, Visakorpi T, Maitland NJ (2015) MicroRNA expression profile of primary prostate cancer stem cells as a source of biomarkers and therapeutic targets. Eur Urol 67(1): 7-10.

Tolcher AW, Messersmith WA, Mikulski SM, Papadopoulos KP, Kwak EL, Gibbon DG, Patnaik A, Falchook GS, Dasari A, Shapiro GI, Boylan JF, Xu ZX, Wang K, Koehler A, Song J, Middleton SA, Deutsch J, Demario M, Kurzrock R, Wheler JJ (2012) Phase I study of RO4929097, a gamma secretase inhibitor of Notch signaling, in patients with refractory metastatic or locally advanced solid tumors. J Clin Oncol 30(19): 2348-2353.

Wu L, Aster JC, Blacklow SC, Lake R, Artavanis-Tsakonas S, Griffin JD (2000) MAML1, a human homologue of Drosophila mastermind, is a transcriptional co-activator for NOTCH receptors. Nat Genet 26(4): $484-489$.

Supplementary Information accompanies this paper on British Journal of Cancer website (http://www.nature.com/bjc) 